\title{
Free Vibration Analysis of an Euler Beam of Variable Width on the Winkler Foundation Using Homotopy Perturbation Method
}

\author{
Utkan Mutman \\ Department of Civil Engineering, Kocaeli University, 41380 Kocaeli, Turkey \\ Correspondence should be addressed to Utkan Mutman; utkanmutman@gmail.com
}

Received 24 February 2013; Accepted 30 March 2013

Academic Editor: Safa Bozkurt Coşkun

Copyright (C) 2013 Utkan Mutman. This is an open access article distributed under the Creative Commons Attribution License, which permits unrestricted use, distribution, and reproduction in any medium, provided the original work is properly cited.

\begin{abstract}
Homotopy Perturbation Method (HPM) is employed to investigate the vibration of an Euler beam resting on an elastic foundation. The beam is assumed to have variable stiffness along its length. HPM is an easy-to-use and very efficient technique for the solution of linear or nonlinear problems. HPM produces analytical approximate expression which is continuous in the solution domain. This work assures that HPM is a promising method for the vibration analysis of the variable stiffness Euler beams on elastic foundation. Different case problems have been solved by using the technique, and solutions have been compared with those available in the literature.
\end{abstract}

\section{Introduction}

In geotechnical engineering, it is widely seen in application that pipelines, shallow foundations, and piles are modeled as a beam in the analysis procedure. There are also various types of foundation models such as Winkler, Pasternak, and Vlasov that have been used in the analysis of structures on elastic foundations.

The most frequently used foundation model in the analysis of beam on elastic foundation problems is the Winkler foundation model. In the Winkler model, the soil is modeled as uniformly distributed, mutually independent, and linear elastic vertical springs which produce distributed reactions in the direction of the deflection of the beam. The winkler model requires a single parameter $k$, representing the soil properties. However, since the model does not take into account either continuity or cohesion of the soil, it may be considered as a rather crude representation of the soil.

As stated before pipelines, shallow foundations and piles can be modeled as a beam. Hence, produced results may find various application areas. There are also different beam types in theory. The well-known is the Euler-Bernoulli beam which is suitable for slender beams. For moderately short and thick beams, the Timoshenko beam model has to be used in the analysis.

Vibration of a constant stiffness Euler beam on elastic foundation was studied previously by Balkaya et al. [1] and Ozturk and Coskun [2]. Balkaya et al. [1] used Differential Transform Method while Ozturk and Coskun [2] used HPM in their studies. Avramidis and Morfidis [3] studied bending of beams on three-parameter elastic foundation. De Rosa [4] analyzed free vibration of Timoshenko beams on two-parameter elastic foundation. Matsunaga [5] studied vibration and buckling of deep beam columns on twoparameter elastic foundations. El-Mously [6] determined fundamental frequencies of the Timoshenko beams mounted on the Pasternak foundation. Chen $[7,8]$ analyzed vibration of beam resting on an elastic foundation by the differential quadrature element method (DQEM). Coşkun [9] investigated the response of a finite beam on a tensionless Pasternak foundation subjected to a harmonic load. Chen et al. [10] developed a mixed method for bending and free vibration of beams resting on a Pasternak elastic foundation. Maheshwari et al. [11] studied the response of beams on a tensionless extensible geosynthetic-reinforced earth bed subjected to moving loads. Auciello and De Rosa [12] developed different 


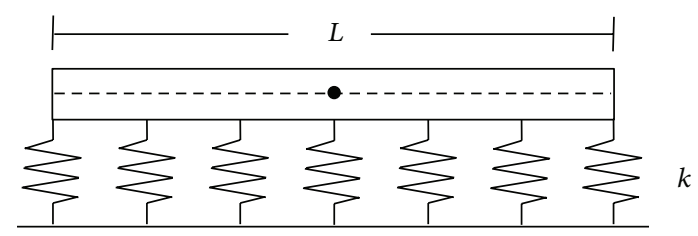

FIGURE 1: Representation of a beam on the Winkler foundation.

approaches to the dynamic analysis of beams on soils subjected to subtangential forces.

\section{The Equations of Motion and Boundary Conditions}

An Euler beam resting on the Winkler foundation shown in Figure 1 is considered in this study. The equation of motion for this problem is given as follows:

$$
\frac{\partial^{2}}{\partial x^{2}}\left(E I(x) \frac{\partial^{2} w}{\partial x^{2}}\right)+k(x) w+\rho A(x)\left(\frac{\partial^{2} w}{\partial t^{2}}\right)=0,
$$

where $k$ is the spring constant, $w$ is deflection, $\rho$ is the mass density, $A$ is the cross-sectional area, EI is the beam stiffness, and $I$ is the area moment of inertia about the neutral axis. The deflection is a function of both space and time, that is, $w=w(x, t)$ in which space variable $x$ is measured along the length of the beam and $t$ represents any particular instant of time.

Due to the support conditions at both ends of the beam, different conditions have to be imposed to the obtained solution to determine unknowns included in final approximation produced by HPM. These conditions are given as follows.

(a) For clamped-clamped beam the end conditions are

$$
w=\frac{\partial w}{\partial x}=0 \quad \text { at } x=0, L
$$

(b) For cantilever beam (clamped-free) the end conditions are

$$
\begin{gathered}
w=\frac{\partial w}{\partial x}=0 \quad \text { at } x=0, \\
\frac{\partial^{2} w}{\partial x^{2}}=\frac{\partial^{3} w}{\partial x^{3}}=0 \quad \text { at } x=L .
\end{gathered}
$$

(c) For simply supported beam (pinned-pinned) the end conditions are

$$
w=\frac{\partial^{2} w}{\partial x^{2}}=0 \quad \text { at } x=0, L .
$$

(d) For clamped-simply supported (pinned) beam the end conditions are

$$
\begin{array}{cl}
w=\frac{\partial w}{\partial x}=0 & \text { at } x=0, \\
w=\frac{\partial^{2} w}{\partial x^{2}}=0 & \text { at } x=L .
\end{array}
$$

Now, free vibration analysis of the beams with variable stiffness resting on elastic foundations will be formulated.

A solution is assumed as the following form to formulate the analysis of the presented problem by the separation of variables

$$
w(x, t)=W(x) e^{i \omega t},
$$

where $\omega$ is the circular frequency for the vibration. Substituting (6) into (1), equations of motion become as follows:

$$
\frac{d^{2}}{d x^{2}}\left(E I(x) \frac{d^{2} W}{d x^{2}}\right)+k W=\rho A \omega^{2} W .
$$

This equation can be rearranged as

$$
\begin{aligned}
\frac{\partial^{4} w}{\partial x^{4}} & +2 \frac{E I(x)^{\prime}}{E I(x)} \frac{\partial^{3} w}{\partial x^{3}}+\frac{E I(x)^{\prime \prime}}{E I(x)} \frac{\partial^{2} w}{\partial x^{2}}+\frac{k(x)}{E I(x)} w \\
& +\frac{\rho A(x)}{E I(x)}\left(\frac{\partial^{2} w}{\partial t^{2}}\right)=0,
\end{aligned}
$$

where $(\cdot)^{\prime}$ denotes total derivative with respect to $x$. The governing equation is now rewritten in a nondimensional form. This procedure is provided from [1] in which a constant stiffness beam was analyzed. The notation is maintained in this study for the comparison purposes. The nondimensional parameters for the Eulerbeam on the Winkler foundation are defined as [1]

$$
\bar{x}=\frac{x}{L}, \quad \bar{W}=\frac{W}{L}, \quad \lambda=\frac{k L^{4}}{E I}, \quad \bar{\omega}=\omega \sqrt{\frac{\rho A}{k}} .
$$

Using these parameters, nondimensional form of the equations and formulation procedures are explained in the following sections.

\section{Homotopy Perturbation Method}

In recent years, efforts towards application of analytical approximate solution techniques for nonlinear problems have increased. In these studies, He's Homotopy Perturbation Method [13-18] can be considered as one of the most promising methods for nonlinear problems. The HPM provides an analytical approximate expression as the solution for the problems which are continuous in the solution domain. The technique is applied to an equation of the form $L(u)+N(u)=$ $f(r), r \in \Omega$ with boundary conditions $B(u, \partial u / \partial n)=0, r \in \Gamma$, where $L$ is a linear operator, $N$ is a nonlinear operator, $B$ is a boundary operator, $\Gamma$ is the boundary of the domain ' $\Omega$, and $f(r)$ is a known analytic function. HPM defines a homotopy as $v(r, p)=\Omega \times[0,1] \rightarrow R$ which satisfies the following inequalities:

$$
\begin{aligned}
H(v, p)= & (1-p)\left[L(v)-L\left(u_{0}\right)\right] \\
& +p[L(v)+N(v)-f(r)]=0
\end{aligned}
$$

or

$$
H(v, p)=L(v)-L\left(u_{0}\right)+p L\left(u_{0}\right)+p[N(v)-f(r)]=0,
$$




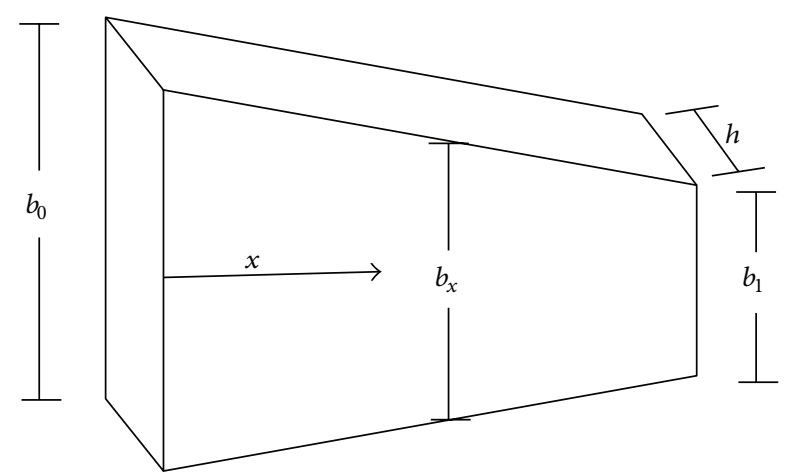

Figure 2: Beam with a linearly varying width.

where $r \in \Omega$ and $p \in[0,1]$ are imbedding parameters, $u_{0}$ is an initial approximation which satisifies the boundary conditions. From (10)-(11), we have

$$
\begin{gathered}
H(v, 0)=L(v)-L\left(u_{0}\right)=0, \\
H(v, 1)=L(v)+N(v)-f(r)=0 .
\end{gathered}
$$

The changing process of $p$ from zero to unity is that of $v(r, p)$ from $u_{0}$ to $u(r)$. In topology, this deformation $L(v)-L\left(u_{0}\right)$ and $L(v)+N(v)-f(r)$ are called homotopic. The method expresses the solution of (10)-(11) as a power series in $p$ as follows:

$$
v=v_{0}+p v_{1}+p^{2} v_{2}+p^{3} v_{3}+\cdots
$$

The approximate solution of $L(u)+N(u)=f(r), r \in \Omega$ can be obtained as

$$
u=\lim _{p \rightarrow 1} v=v_{0}+v_{1}+v_{2}+\cdots
$$

The convergence of the series in (14) has been proven in [13-18].

\section{HPM Formulation and Solution Procedure for Presented Problem}

In this study, a variable stiffness is assumed for the beam considered. The variation is applied by changing the width of the beam along its length. Two different variations in stiffness are assumed. These are linear and exponential variations.

In the linear variation, beam width varies in a linear manner along the beam's length which is shown in Figure 2.

The variable width is formulated as

$$
b(x)=b_{0}(1-\alpha x)
$$

where the dimension of $\alpha$ is $[1 / L]$. For the exponential variation, beam width varies exponentially along the beam's length as shown in Figure 3.

Variable width of the beam in this case is

$$
b(x)=b_{0} e^{-\alpha x}
$$

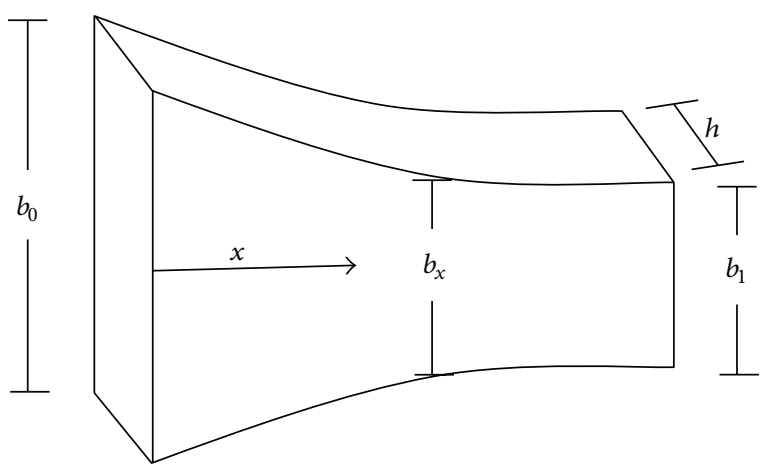

FIgURE 3: Beam with an exponentially varying width.

where $\alpha$ has the same dimension as in linear case. By the use of variable width, both cross-sectional area and moment of inertia are variable,

$$
\begin{aligned}
& A(x)=b(x) h, \\
& I(x)=b(x) \frac{h^{3}}{12} .
\end{aligned}
$$

In the following sections, formulation procedures are given separately for each case.

4.1. Formulation for Linear Variation. Employing (15) into (17), variable cross-sectional areas and variable stiffness become

$$
\begin{gathered}
A(x)=b_{0} h(1-\alpha x)=A_{0}(1-\alpha x), \\
E I(x)=E b_{0} \frac{h^{3}}{12}(1-\alpha x)=E I_{0}(1-\alpha x),
\end{gathered}
$$

where $A_{0}$ and $I_{0}$ are the cross-sectional area and moment of inertia of the section at the origin, respectively. Inserting (18) into (8)

$$
\begin{gathered}
\frac{\partial^{4} w}{\partial x^{4}}-\frac{2 \alpha E I_{0}}{E I_{0}(1-\alpha x)} \frac{\partial^{3} w}{\partial x^{3}}+\frac{k(x)}{E I_{0}(1-\alpha x)} w \\
+\frac{\rho A_{0}}{E I_{0}}\left(\frac{\partial^{2} w}{\partial t^{2}}\right)=0 .
\end{gathered}
$$

This equation can be rewritten as

$$
\begin{gathered}
\frac{\partial^{4} w}{\partial x^{4}}-2 \alpha\left(\frac{1}{1-\alpha x}\right) \frac{\partial^{3} w}{\partial x^{3}}+\frac{k(x)}{E I_{0}}\left(\frac{1}{1-\alpha x}\right) w \\
+\frac{\rho A_{0}}{E I_{0}}\left(\frac{\partial^{2} w}{\partial t^{2}}\right)=0 .
\end{gathered}
$$

Employing (6)

$$
W^{i v}-2 \alpha \xi(x) W^{\prime \prime \prime}+\frac{k(x)}{E I_{0}} \xi(x) W-\frac{\rho A_{0}}{E I_{0}} \omega^{2} W=0 .
$$


Equation (21) can be made nondimensional in view of (9) as follows:

$$
\bar{W}^{i v}-2 \bar{\alpha} \xi(\bar{x}) \bar{W}^{\prime \prime \prime}+\lambda\left(\xi(\bar{x})-\bar{\omega}^{2}\right) W=0,
$$

where

$$
\begin{gathered}
\bar{x}=\frac{x}{L}, \quad \bar{W}=\frac{W}{L}, \quad \lambda=\frac{k L^{4}}{E I_{0}}, \quad \bar{\omega}=\omega \sqrt{\frac{\rho A_{0}}{k}}, \\
\xi(\bar{x})=\frac{1}{1-\bar{\alpha} \bar{x}}, \quad \bar{\alpha}=\alpha L .
\end{gathered}
$$

By the application of HPM, the following iteration algorithm is obtained:

$$
\begin{gathered}
\bar{W}_{0}^{i v}-u_{0}^{i v}=0, \\
\bar{W}_{1}^{i v}+u_{0}^{i v}-2 \bar{\alpha} \xi(\bar{x}) \bar{W}_{0}^{\prime \prime \prime}+\lambda\left(\xi(\bar{x})-\bar{\omega}^{2}\right) \bar{W}_{0}=0, \\
\bar{W}_{n}^{i v}-2 \bar{\alpha} \xi(\bar{x}) \bar{W}_{n-1}^{\prime \prime \prime}+\lambda\left(\xi(\bar{x})-\bar{\omega}^{2}\right) \bar{W}_{n-1}=0, \quad n \geq 2 .
\end{gathered}
$$

4.2. Formulation for Exponential Variation. Employing (16) into (17), variable cross-sectional areas and variable stiffness become

$$
\begin{gathered}
A(x)=b_{0} h e^{-\alpha x}=A_{0} e^{-\alpha x}, \\
E I(x)=E \frac{h^{3}}{12} b_{0} e^{-\alpha x}=E I_{0} e^{-\alpha x} .
\end{gathered}
$$

Inserting (25) into (8)

$\frac{\partial^{4} w}{\partial x^{4}}-2 \alpha \frac{\partial^{3} w}{\partial x^{3}}+\alpha^{2} \frac{\partial^{2} w}{\partial x^{2}}+\frac{k(x)}{E I_{0} e^{-\alpha x}} w+\frac{\rho A_{0}}{E I_{0}}\left(\frac{\partial^{2} w}{\partial t^{2}}\right)=0$.

Employing (6)

$$
W^{i v}-2 \alpha W^{\prime \prime \prime}+\alpha^{2} W^{\prime \prime}+\frac{k(x)}{E I_{0}} e^{\alpha x} W-\frac{\rho A_{0}}{E I_{0}} \omega^{2} W=0 .
$$

Equation (21) can be made nondimensional in view of (9) as follows:

$$
\bar{W}^{i v}-2 \bar{\alpha} \bar{W}^{\prime \prime \prime}+\bar{\alpha}^{2} \bar{W}^{\prime \prime}+\lambda\left(e^{\bar{\alpha} \bar{x}}-\bar{\omega}^{2}\right) W=0 .
$$

By the application of HPM, the following iteration algorithm is obtained:

$$
\begin{gathered}
\bar{W}_{0}^{i v}-u_{0}^{i v}=0, \\
\bar{W}_{1}^{i v}+u_{0}^{i v}-2 \bar{\alpha} \bar{W}_{0}^{\prime \prime \prime}+\bar{\alpha}^{2} \bar{W}_{0}^{\prime \prime}+\lambda\left(e^{\bar{\alpha} \bar{x}}-\bar{\omega}^{2}\right) \bar{W}_{0}=0, \\
\bar{W}_{n}^{i v}-2 \bar{\alpha} \bar{W}_{n-1}^{\prime \prime \prime}+\bar{\alpha}^{2} \bar{W}_{n-1}^{\prime \prime}+\lambda\left(e^{\bar{\alpha} \bar{x}}-\bar{\omega}^{2}\right) \bar{W}_{n-1}=0,
\end{gathered}
$$

4.3. Solution Procedure. An initial approximation may be chosen as a cubic polynomial with four unknown coefficients. There exist four boundary conditions, that is, two at each end of the column, in the presented problem. Hence, an initial approximation of the following choice may be employed:

$$
\bar{W}_{0}=A \bar{x}^{3}+B \bar{x}^{2}+C \bar{x}+D \text {. }
$$

In the computations, twenty iterations are conducted and four boundary conditions for each case are rewritten by using the final approximation of iteration. Each boundary condition produces an equation containing four unknowns spread from the initial approximation. These nondimensional boundary conditions are as follows.

Clamped-clamped beam:

$$
\bar{W}=\frac{d \bar{W}}{d \bar{x}}=0 \quad \text { at } \bar{x}=0,1 .
$$

Clamped-free (cantilever) beam:

$$
\begin{gathered}
\bar{W}=\frac{d \bar{W}}{d \bar{x}}=0 \quad \text { at } \bar{x}=0, \\
\frac{d^{2} \bar{W}}{d \bar{x}^{2}}=\frac{d^{3} \bar{W}}{d \bar{x}^{3}}=0 \quad \text { at } \bar{x}=1 .
\end{gathered}
$$

Pinned-pinned (simply supported) beam:

$$
\bar{W}=\frac{d^{2} \bar{W}}{d \bar{x}^{2}}=0 \quad \text { at } \bar{x}=0,1
$$

Clamped-pinned beam:

$$
\begin{array}{ll}
\bar{W}=\frac{d \bar{W}}{d \bar{x}}=0 \quad \text { at } \bar{x}=0, \\
\bar{W}=\frac{d^{2} \bar{W}}{d \bar{x}^{2}}=0 \quad \text { at } \bar{x}=1 .
\end{array}
$$

Hence, four equations in four unknowns may be written with respect to the boundary conditions of the problem. These equations can be represented in matrix form as follows:

$$
[M(\bar{\omega})]\{A\}=\{0\},
$$

where $\{A\}=\langle A B C D\rangle^{T}$. For a nontrivial solution, determinant of coefficient matrix must be zero. Determinant of coefficient matrix yields a characteristic equation in terms of $\bar{\omega}$. Positive real roots of this equation are the normalized free vibration frequencies for the case considered.

\section{Numerical Results}

5.1. Constant Stiffness Case. As the first example, Euler beam of constant stiffness (i.e., EI is constant) with different boundary conditions is investigated. For the sake of comparison, all the values are set to unity such as $I=E=A=\rho=1$; hence, $\lambda=1$, according to previous studies [7]. Both algorithms given for linear and exponential variations lead to constant stiffness when $\alpha=0$. 
TABLE 1: Normalized free vibration frequencies of simply supported beam resting on the Winkler foundation.

\begin{tabular}{|c|c|c|c|c|c|}
\hline Method & $\omega_{1}$ & $\omega_{2}$ & $\omega_{3}$ & $\omega_{4}$ & $\omega_{5}$ \\
\hline HPM & 9.92014 & 39.4911 & 88.8321 & 157.9168 & 246.7421 \\
\hline DTM [1] & 9.92014 & 39.4911 & 88.8321 & - & - \\
\hline DQEM [7] & 9.92014 & 39.4913 & 89.4002 & - & - \\
\hline Exact solution [1] & 9.92014 & 39.4911 & 88.8321 & - & - \\
\hline
\end{tabular}

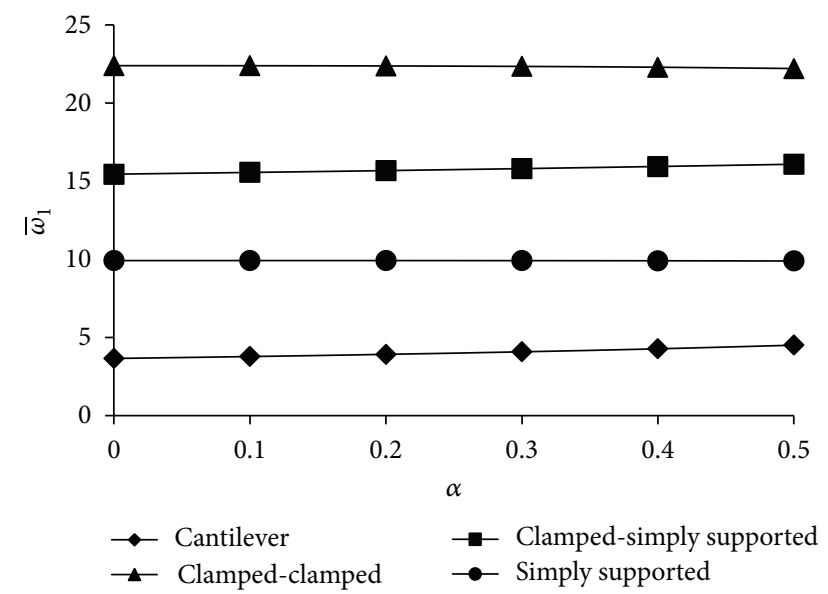

FIGURE 4: Variation of normalized first mode frequency with respect to normalized variation coefficient (linear variation in stiffness).

In Table 1 , first three normalized free vibration frequencies for simply supported (pinned-pinned) beam are compared with previous available results in the literature and the exact solution. Excellent agreement is observed with the exact solution.

The first five natural frequencies for clamped-clamped beam and cantilever (clamped-free) beam are presented in Tables 2 and 3, respectively. They are compared with the available solutions.

Excellent agreement is observed with previous available results for both cantilever and clamped-clamped beams. Clamped-pinned beam was not included in the studies used for comparison. Hence, only the result obtained from HPM is tabulated for this case in Table 4.

As one can see, perfect agreement is obtained for constant stiffness case. This issue is mainly due to constant coefficient governing equation. In the following sections, variable stiffness cases are investigated.

5.2. Linearly Varying Stiffness. Linearly varying beam width results in a linearly varying flexural stiffness. The variation is based on parameter $\alpha$. A number of case studies are conducted with respect to parameter $\alpha$, and the results are given in Tables 5, 6, 7, and 8 .

Variation of normalized free vibration frequencies $(\bar{\omega})$ with respect to nondimensional variation parameter $\bar{\alpha}$ for each beam are also given in Figures 4, 5, 6, 7, and 8.

5.3. Exponentially Varying Stiffness. Exponentially varying beam width results in an exponentially varying flexural

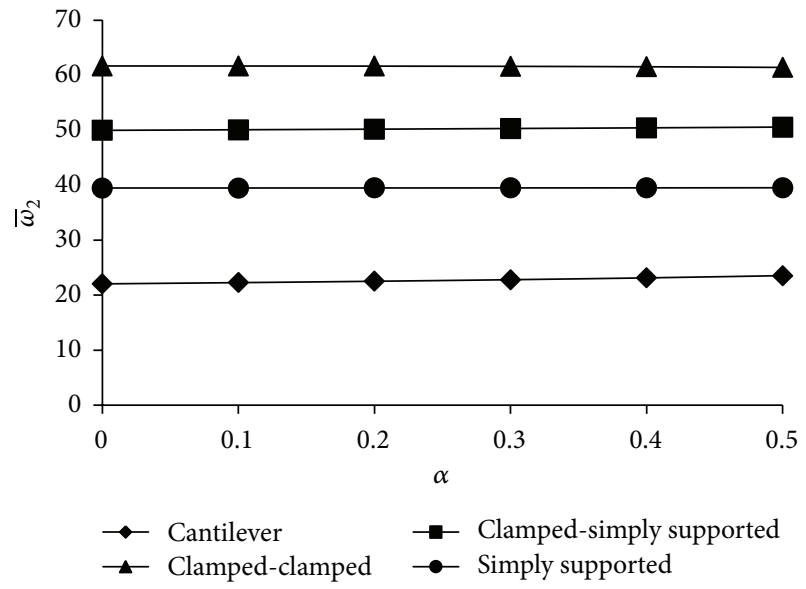

FIGURE 5: Variation of normalized second mode frequency with respect to normalized variation coefficient (linear variation in stiffness).

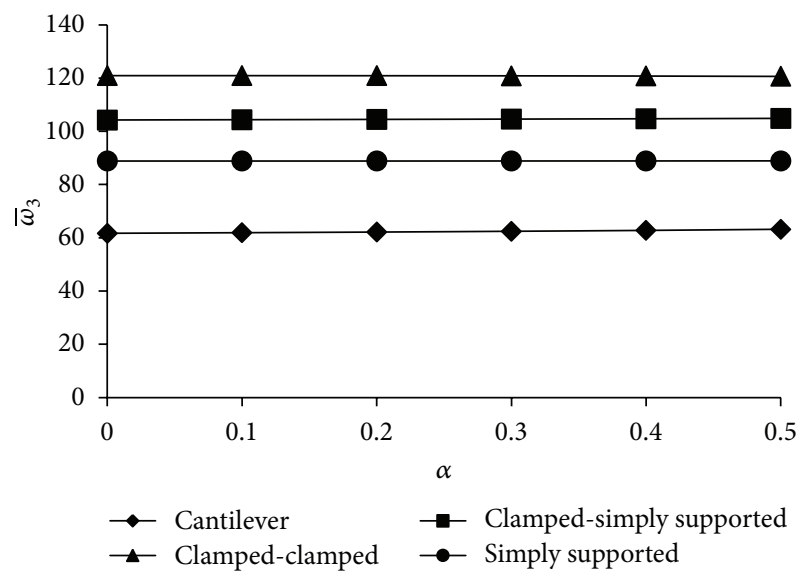

FIGURE 6: Variation of normalized third mode frequency with respect to normalized variation coefficient (linear variation in stiffness).

stiffness. The variation is again based on parameter $\alpha$, and a number of case studies are also conducted with respect to parameter $\alpha$. Results are given in Tables 9, 10, 11, and 12 .

Variation of normalized free vibration frequencies $(\bar{\omega})$ with respect to nondimensional variation parameter $\bar{\alpha}$ for each beam are also given in Figures 9, 10, 11, 12, and 13. 
TABLE 2: Normalized free vibration frequencies of clamped-clamped beam resting on the Winkler foundation.

\begin{tabular}{lcccrr}
\hline Method & $\omega_{1}$ & $\omega_{2}$ & $\omega_{3}$ & $\omega_{4}$ & $\omega_{5}$ \\
\hline HPM & 22.3956 & 61.6809 & 120.908 & 199.862 & 298.557 \\
DTM [1] & 22.3733 & 61.6728 & 120.903 & 199.859 & 298.556 \\
DQEM [7] & 22.3956 & 61.6811 & 120.910 & 199.885 & 298.675 \\
\hline
\end{tabular}

TABLE 3: Normalized free vibration frequencies of cantilever beam resting on the Winkler foundation.

\begin{tabular}{|c|c|c|c|c|c|}
\hline Method & $\omega_{1}$ & $\omega_{2}$ & $\omega_{3}$ & $\omega_{4}$ & $\omega_{5}$ \\
\hline HPM & 3.65546 & 22.0572 & 61.7053 & 120.906 & 199.862 \\
\hline DTM [1] & 3.65546 & 22.0572 & 61.7053 & 120.906 & 199.862 \\
\hline DQEM [7] & 3.65544 & 22.0572 & 61.7057 & 120.911 & 199.894 \\
\hline
\end{tabular}

TABLE 4: Normalized free vibration frequencies of clamped-pinned beam resting on the Winkler foundation.

\begin{tabular}{lccccc}
\hline Method & $\omega_{1}$ & $\omega_{2}$ & $\omega_{3}$ & $\omega_{4}$ & $\omega_{5}$ \\
\hline HPM & 15.451 & 49.975 & 104.253 & 178.273 & 272.033 \\
\hline
\end{tabular}

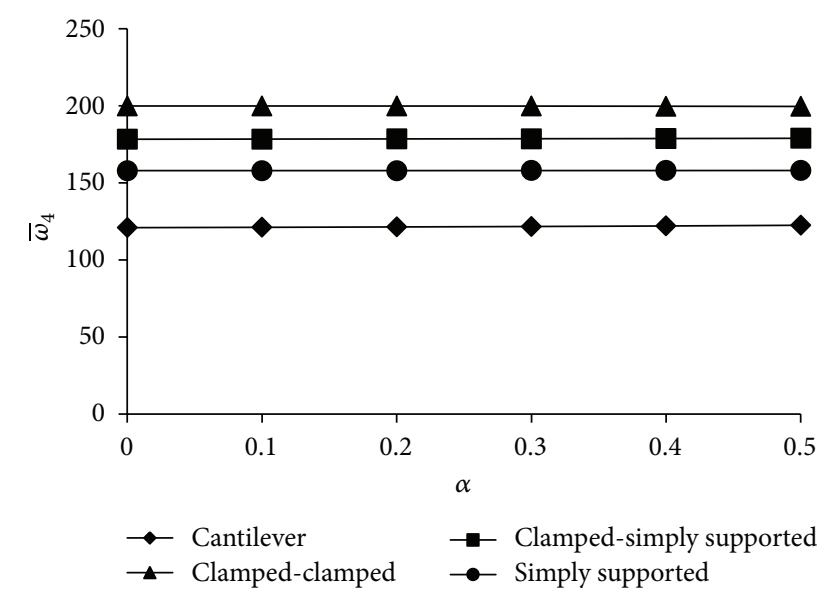

FIGURE 7: Variation of normalized fourth mode frequency with respect to normalized variation coefficient (linear variation in stiffness).

\section{Conclusions}

In this study, HPM is introduced for the free vibration analysis of variable stiffness Euler beams on elastic foundations. As a demonstration of application of the method, firstly the case studies for which previous results were available are chosen. In these studies, constant stiffness Euler beams were considered, and first analyses are conducted for constant stiffness case for comparison with the available results. HPM produced results which are in excellent agreement with the previously available solutions that encourage the application of the method for variable stiffness beams. To represent a variation in stiffness, a rectangular beam with varying width is considered, and two types of variation are taken into consideration. These are, namely, linear and exponential changes. Such varying widths lead to linearly and exponentially varying stiffnesses. The analyses are expanded

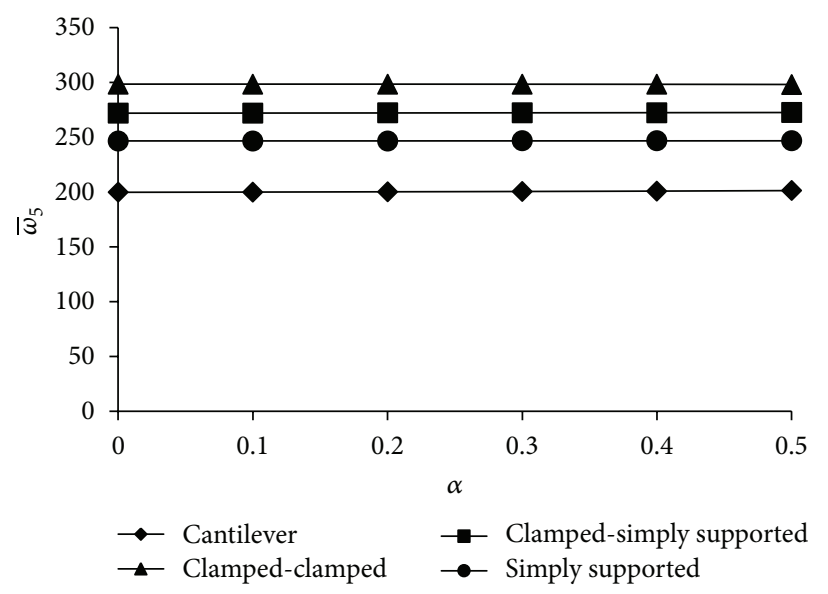

FIGURE 8: Variation of normalized fifth mode frequency with respect to normalized variation coefficient (linear variation in stiffness).

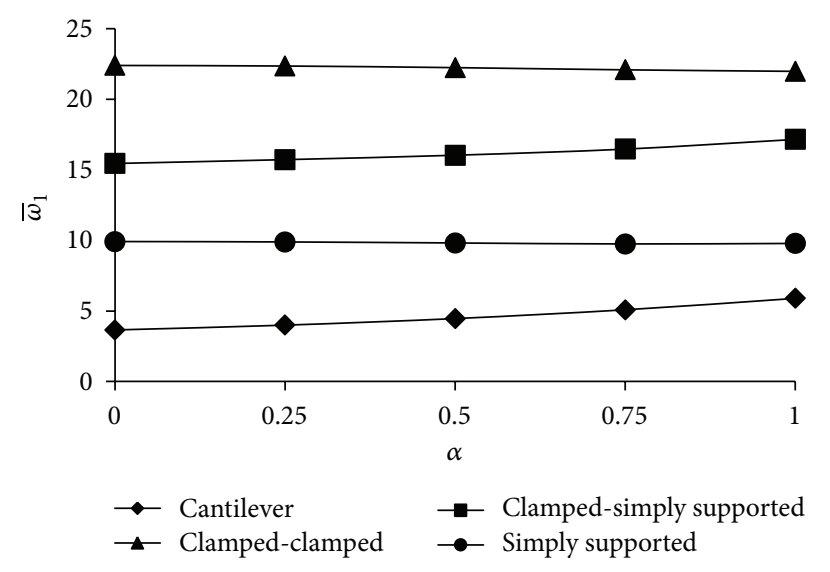

FIGURE 9: Variation of normalized first mode frequency with respect to normalized variation coefficient (exponential variation in stiffness).

for variable stiffness cases. HPM also produced reasonable results for the vibration of variable stiffness beams which show the efficiency of the method. For the variable stiffness problems, the governing equation is a differential equation with variable coefficients, and it is not easy to obtain analytical 
TABLE 5: Normalized free vibration frequencies of cantilever beam resting on Winkler foundation with linearly varying flexural stiffness.

\begin{tabular}{lcccccc}
\hline$\alpha$ & 0.00 & 0.10 & 0.20 & 0.30 & 0.40 & 0.50 \\
\hline$\omega_{1}$ & 3.65546 & 3.77785 & 3.91814 & 4.08113 & 4.27363 & 2.50571 \\
$\omega_{2}$ & 22.0572 & 22.2779 & 22.5271 & 22.8129 & 62.4458 & 62.7867 \\
$\omega_{3}$ & 61.7053 & 61.9182 & 62.1616 & 121.6528 & 122.0024 & 63.2104 \\
$\omega_{4}$ & 120.9061 & 121.1194 & 121.3645 & 200.6117 & 200.9661 \\
$\omega_{5}$ & 199.8620 & 200.0755 & 200.3213 & & 122.4434 \\
\hline
\end{tabular}

TABLE 6: Normalized free vibration frequencies of clamped-pinned beam resting on the Winkler foundation with linearly varying flexural stiffness.

\begin{tabular}{lcccccc}
\hline$\alpha$ & 0.00 & 0.10 & 0.20 & 0.30 & 0.40 & 0.50 \\
\hline$\omega_{1}$ & 15.4506 & 15.5615 & 15.6801 & 15.8069 & 15.9427 & 50.4316 \\
$\omega_{2}$ & 49.9749 & 50.0781 & 50.1878 & 50.3052 & 104.0879 \\
$\omega_{3}$ & 104.2525 & 104.3556 & 104.4655 & 104.5836 & 170.5685 \\
$\omega_{4}$ & 178.2725 & 178.3756 & 178.4853 & 178.6036 & 178.7333 & 104.8544 \\
$\omega_{5}$ & 272.0328 & 272.1358 & 272.2455 & 272.3640 & 272.4944 & 178.8785 \\
\hline
\end{tabular}

TABLE 7: Normalized free vibration frequencies of clamped-clamped beam resting on the Winkler foundation with linearly varying flexural stiffness.

\begin{tabular}{lcccccc}
\hline$\alpha$ & 0.00 & 0.10 & 0.20 & 0.30 & 0.40 & 0.50 \\
\hline$\omega_{1}$ & 22.3956 & 22.3922 & 22.3777 & 22.3477 & 22.2958 & 61.5380 \\
$\omega_{2}$ & 61.6809 & 61.6752 & 61.6541 & 61.6114 & 120.8302 & 120.7485 \\
$\omega_{3}$ & 120.9075 & 120.9009 & 120.8775 & 199.7803 & 199.6939 \\
$\omega_{4}$ & 199.8620 & 199.8549 & 199.8302 & 298.4729 & 298.3834 \\
$\omega_{5}$ & 298.5572 & 298.5499 & 298.5243 & & 120.6144 \\
\hline
\end{tabular}

TABLE 8: Normalized free vibration frequencies of simply supported beam resting on the Winkler foundation with linearly varying flexural stiffness.

\begin{tabular}{lcccccc}
\hline$\alpha$ & 0.00 & 0.10 & 0.20 & 0.30 & 0.40 & 0.50 \\
\hline$\omega_{1}$ & 9.9201 & 9.9217 & 9.9210 & 9.9170 & 9.9084 & 9.8932 \\
$\omega_{2}$ & 39.4911 & 39.4928 & 39.4970 & 39.5047 & 39.5166 & 39.5340 \\
$\omega_{3}$ & 88.8321 & 88.8340 & 88.8398 & 88.8511 & 88.8697 & 88.8986 \\
$\omega_{4}$ & 157.9168 & 157.9189 & 157.9257 & 157.9389 & 157.9612 & 157.9966 \\
$\omega_{5}$ & 246.7421 & 246.7443 & 246.7516 & 246.7661 & 246.7907 & 246.8302 \\
\hline
\end{tabular}

TABLE 9: Normalized free vibration frequencies of cantilever beam resting on the Winkler foundation with exponentially varying flexural stiffness.

\begin{tabular}{lccccc}
\hline$\alpha$ & 0.00 & 0.25 & 0.50 & 0.75 & 5.00 \\
\hline$\omega_{1}$ & 3.65546 & 3.99564 & 4.46148 & 5.08383 & 24.85198 \\
$\omega_{2}$ & 22.0572 & 22.6410 & 23.4038 & 64.1348 & 25.9407 \\
$\omega_{3}$ & 61.7053 & 62.2689 & 63.0286 & 123.3890 & 65.8187 \\
$\omega_{4}$ & 120.9061 & 121.4706 & 122.2407 & 202.3738 & 125.1956 \\
$\omega_{5}$ & 199.8620 & 200.4266 & 201.2020 & 204.2517 \\
\hline
\end{tabular}

TABLE 10: Normalized free vibration frequencies of clamped-pinned beam resting on the Winkler foundation with exponentially varying flexural stiffness.

\begin{tabular}{lccccc}
\hline$\alpha$ & 0.00 & 0.25 & 0.50 & 0.75 & 1.00 \\
\hline$\omega_{1}$ & 15.4506 & 15.7228 & 16.0355 & 16.4684 & 5.1543 \\
$\omega_{2}$ & 49.9749 & 50.2233 & 50.5095 & 50.9401 & 1.7103 \\
$\omega_{3}$ & 104.2525 & 104.4999 & 104.7874 & 105.2357 & 106.0716 \\
$\omega_{4}$ & 178.2725 & 178.5190 & 178.8070 & 179.2648 & 180.1380 \\
$\omega_{5}$ & 272.0328 & 272.2787 & 272.5671 & 273.0313 & 273.9289 \\
\hline
\end{tabular}


TABLE 11: Normalized free vibration frequencies of clamped-clamped beam resting on the Winkler foundation with exponentially varying flexural stiffness.

\begin{tabular}{lccccc}
\hline$\alpha$ & 0.00 & 0.25 & 0.50 & 0.75 & 21.00 \\
\hline$\omega_{1}$ & 22.3956 & 22.3562 & 22.2409 & 22.0853 & 61.2498 \\
$\omega_{2}$ & 61.6809 & 61.6245 & 61.4646 & 120.4372 & 61.1004 \\
$\omega_{3}$ & 120.9075 & 120.8450 & 120.6698 & 199.3705 & 120.2857 \\
$\omega_{4}$ & 199.8620 & 199.7961 & 199.6124 & 298.0528 & 199.2211 \\
$\omega_{5}$ & 298.5572 & 298.4893 & 298.3002 & 297.9060 \\
\hline
\end{tabular}

TABLE 12: Normalized free vibration frequencies of simply supported beam resting on the Winkler foundation with exponentially varying flexural stiffness.

\begin{tabular}{lccccc}
\hline$\alpha$ & 0.00 & 0.25 & 0.50 & 0.75 & 9.00 \\
\hline$\omega_{1}$ & 9.9201 & 9.8928 & 9.8179 & 9.7493 & 3.7849 \\
$\omega_{2}$ & 39.4911 & 39.4736 & 39.4584 & 88.546 & 39.9202 \\
$\omega_{3}$ & 88.8321 & 88.8182 & 88.8204 & 158.0782 & 89.4478 \\
$\omega_{4}$ & 157.9168 & 157.9048 & 157.9164 & 246.9262 & 158.6327 \\
$\omega_{5}$ & 246.7421 & 246.7312 & 246.7485 & 247.5200 \\
\hline
\end{tabular}

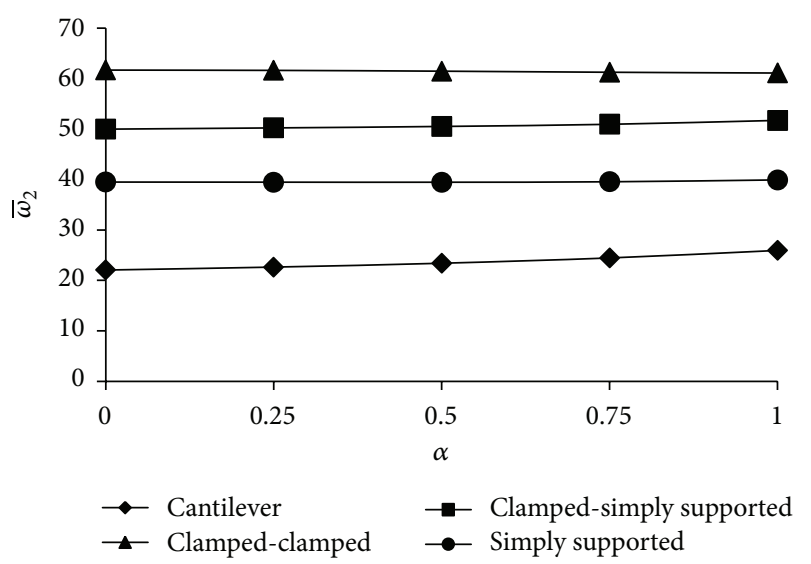

FIGURE 10: Variation of normalized second mode frequency with respect to normalized variation coefficient (exponential variation in stiffness).

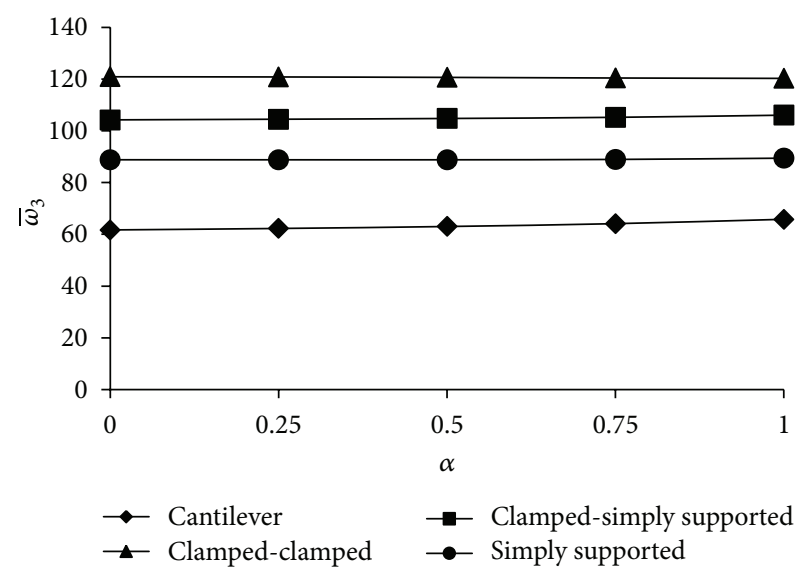

FIGURE 11: Variation of normalized third mode frequency with respect to normalized variation coefficient (exponential variation in stiffness).

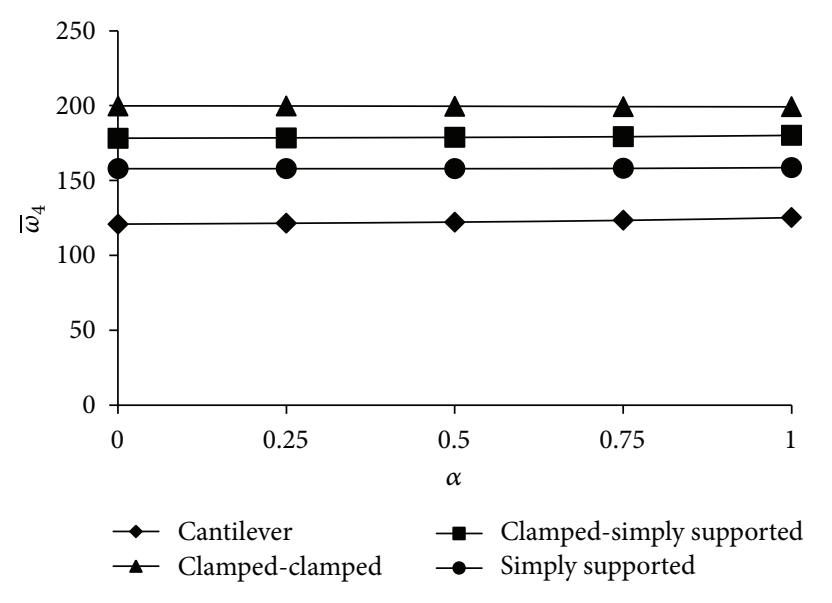

FIGURE 12: Variation of normalized fourth mode frequency with respect to normalized variation coefficient (exponential variation in stiffness).

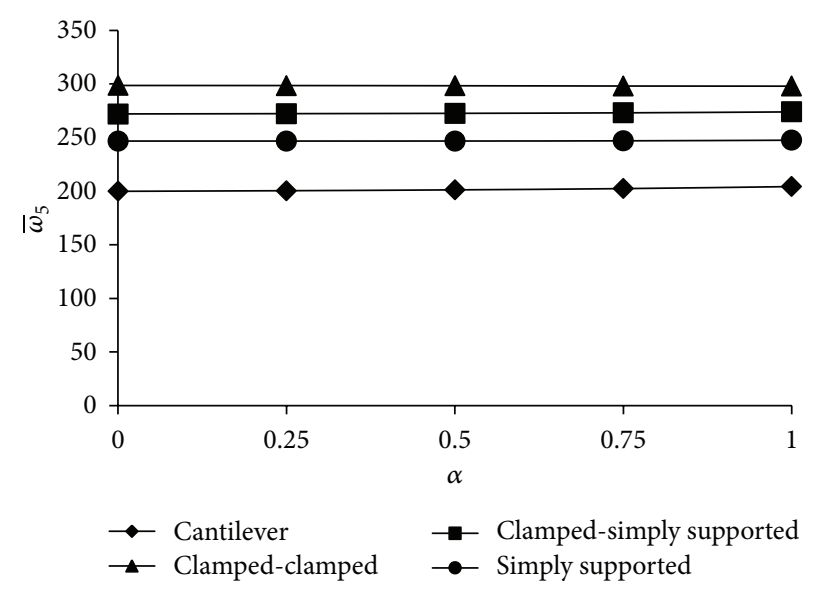

FIGURE 13: Variation of normalized fifth mode frequency with respect to normalized variation coefficient (exponential variation in stiffness). 
solutions for these types of problems. However, it is easy to put those variable parameters into the iteration algorithm of HPM, and the results can be obtained after performing some iterations with the method. The results obtained in this study point out that the proposed method is a powerful and reliable method in the analysis of the presented problem.

\section{References}

[1] M. Balkaya, M. O. Kaya, and A. Sağlamer, "Analysis of the vibration of an elastic beam supported on elastic soil using the differential transform method," Archive of Applied Mechanics, vol. 79, no. 2, pp. 135-146, 2009.

[2] B. Ozturk and S. B. Coskun, "The Homotopy Perturbation Method for free vibration analysis of beam on elastic foundation," Structural Engineering and Mechanics, vol. 37, no. 4, pp. 415-425, 2011.

[3] I. E. Avramidis and K. Morfidis, "Bending of beams on threeparameter elastic foundation," International Journal of Solids and Structures, vol. 43, no. 2, pp. 357-375, 2006.

[4] M. A. De Rosa, "Free vibrations of Timoshenko beams on twoparameter elastic foundation," Computers and Structures, vol. 57, no. 1, pp. 151-156, 1995.

[5] H. Matsunaga, "VIbration and buckling of deep beam-columns on two-parameter elastic foundations," Journal of Sound and Vibration, vol. 228, no. 2, pp. 359-376, 1999.

[6] M. El-Mously, "Fundamental frequencies of Timoshenko beams mounted on Pasternak foundation," Journal of Sound and Vibration, vol. 228, no. 2, pp. 452-457, 1999.

[7] C. N. Chen, "Vibration of prismatic beam on an elastic foundation by the differential quadrature element method," Computers and Structures, vol. 77, no. 1, pp. 1-9, 2000.

[8] C. N. Chen, "DQEM vibration analyses of non-prismatic shear deformable beams resting on elastic foundations," Journal of Sound and Vibration, vol. 255, no. 5, pp. 989-999, 2002.

[9] I. Coşkun, "The response of a finite beam on a tensionless Pasternak foundation subjected to a harmonic load," European Journal of Mechanics, A/Solids, vol. 22, no. 1, pp. 151-161, 2003.

[10] W. Q. Chen, C. F. Lü, and Z. G. Bian, "A mixed method for bending and free vibration of beams resting on a Pasternak elastic foundation," Applied Mathematical Modelling, vol. 28, no. 10, pp. 877-890, 2004.

[11] P. Maheshwari, S. Chandra, and P. K. Basudhar, "Response of beams on a tensionless extensible geosynthetic-reinforced earth bed subjected to moving loads," Computers and Geotechnics, vol. 31, no. 7, pp. 537-548, 2004.

[12] N. M. Auciello and M. A. De Rosa, "Two approaches to the dynamic analysis of foundation beams subjected to subtangential forces," Computers and Structures, vol. 82, no. 6, pp. 519-524, 2004.

[13] J. H. He, "Coupling method of a homotopy technique and a perturbation technique for non-linear problems," International Journal of Non-Linear Mechanics, vol. 35, no. 1, pp. 37-43, 2000.

[14] J. H. He, "The homotopy perturbation method for nonlinear oscillators with discontinuities," Applied Mathematics and Computation, vol. 151, no. 1, pp. 287-292, 2004.

[15] J. H. He, "Application of homotopy perturbation method to nonlinear wave equations," Chaos, Solitons \& Fractals, vol. 26, no. 3, pp. 695-700, 2005.
[16] J. H. He, "Asymptotology by homotopy perturbation method," Applied Mathematics and Computation, vol. 156, no. 3, pp. 591596, 2004.

[17] J.H. He, "Homotopy perturbation method for solving boundary value problems," Physics Letters A, vol. 350, no. 1-2, pp. 87-88, 2006.

[18] J. H. He, "Limit cycle and bifurcation of nonlinear problems," Chaos, Solitons \& Fractals, vol. 26, no. 3, pp. 827-833, 2005. 


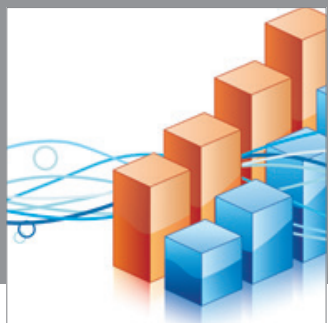

Advances in

Operations Research

mansans

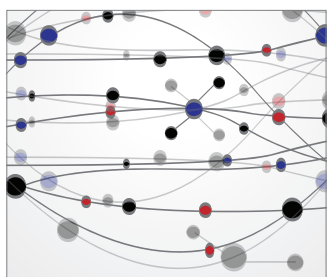

The Scientific World Journal
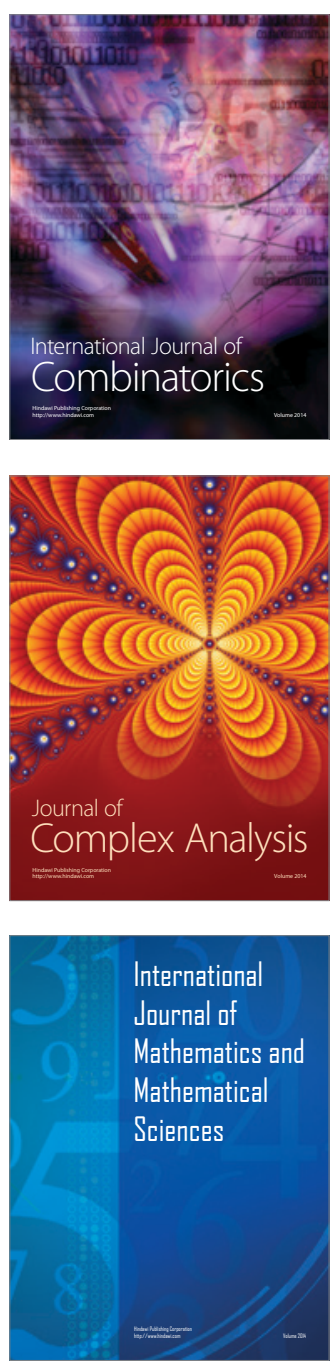
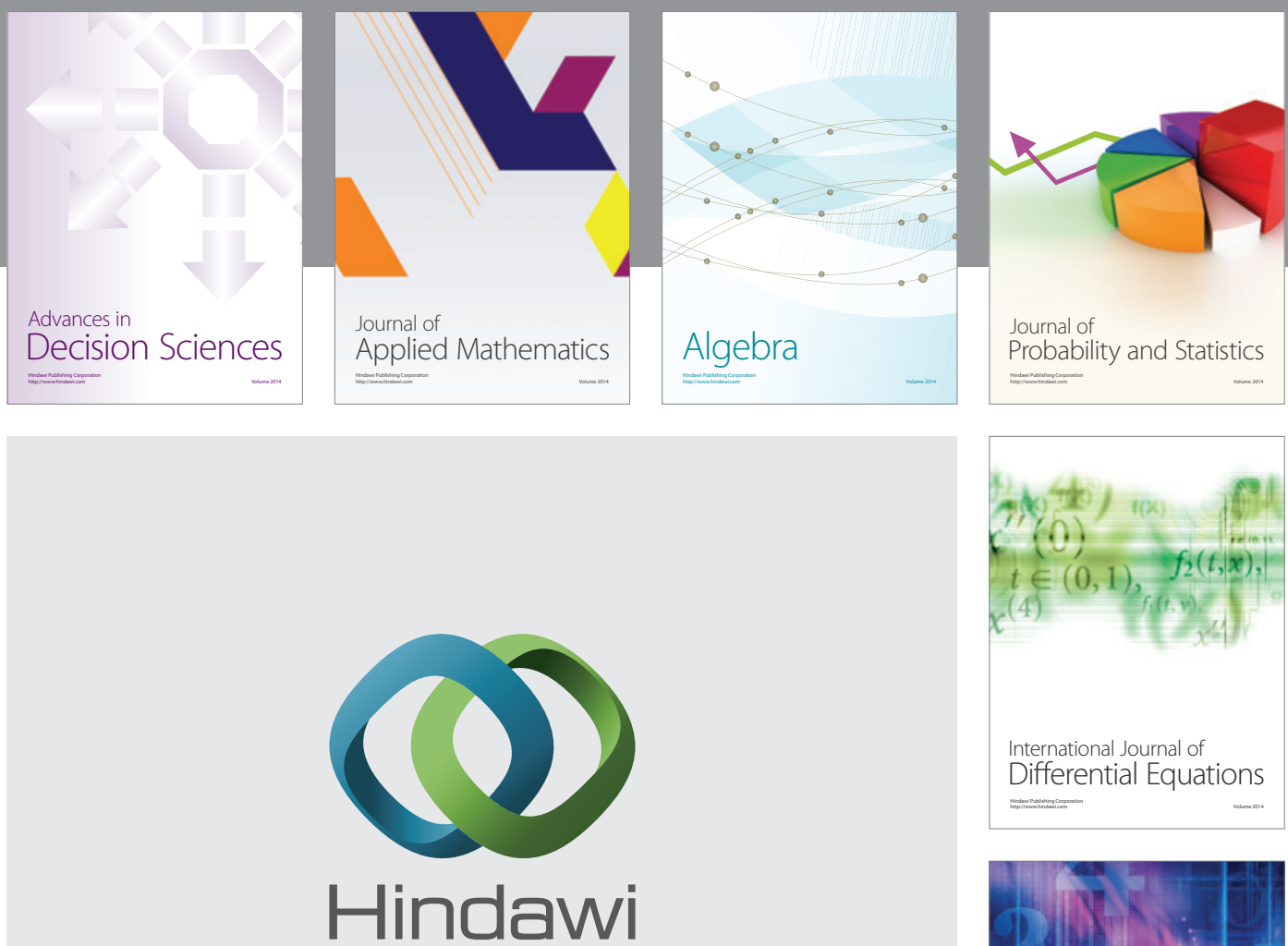

Submit your manuscripts at http://www.hindawi.com
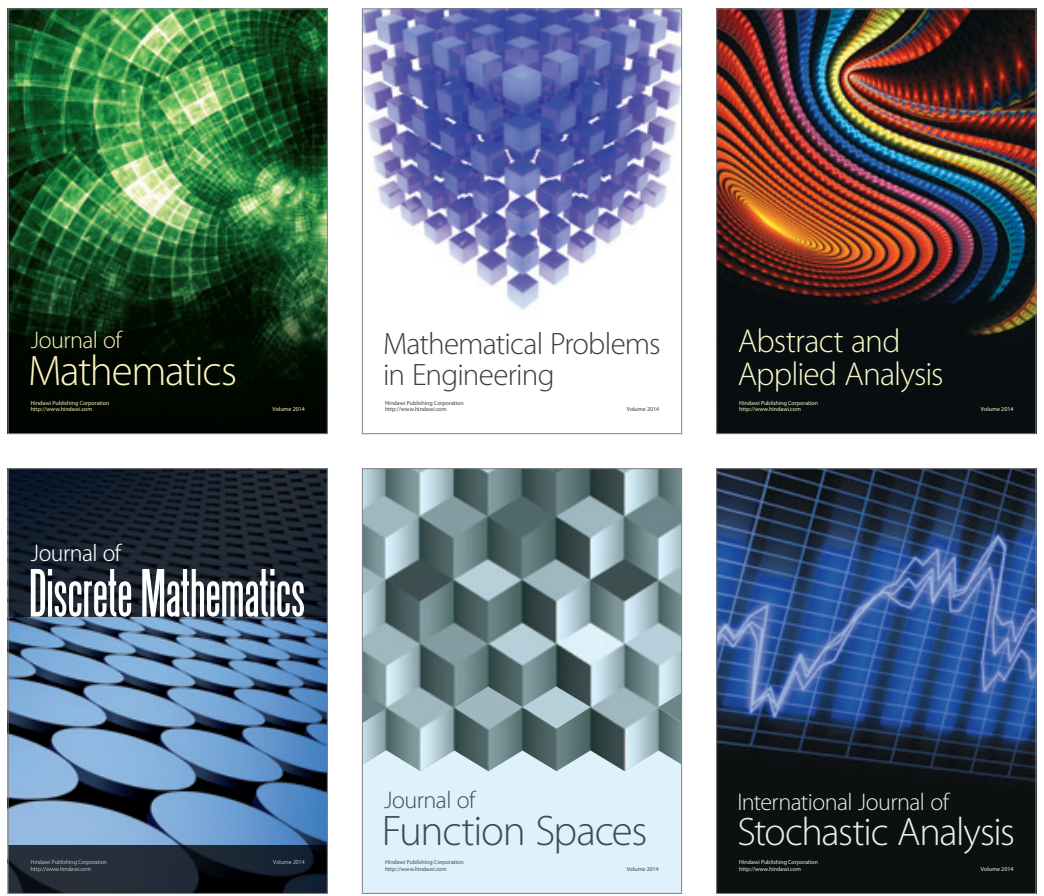

Journal of

Function Spaces

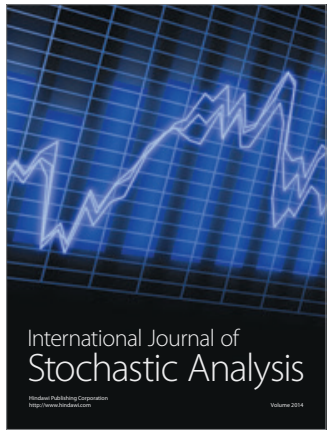

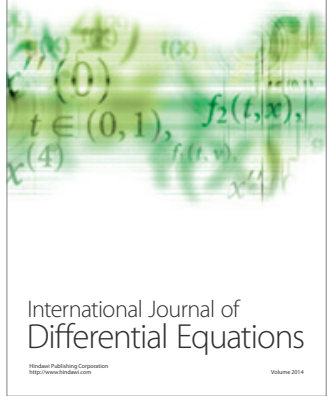
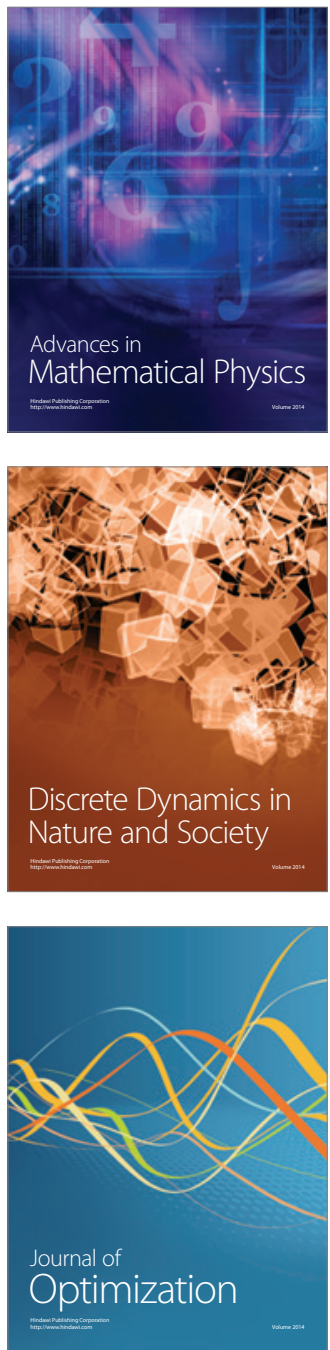